\title{
Quantitative immunohistochemical expression of c Kit in breast carcinomas is predictive of patients' outcome
}

\author{
C Charpin*,', S Giusiano', S Charfi', V Secq', S Carpentier', L Andrac', M-N Lavaut', C Allasia', P Bonnier² and \\ S Garcia'
}

'Department of Pathology, Hôpital Nord and Université de la Méditerranée (Aix Marseille II), Marseille, France; ${ }^{2}$ Department of Gynecologic Oncology, Hôpital de la Conception, Marseille, France

BACKGROUND: c Kit (CDII7) expression in tissues has been reported as a relevant target for specific therapy in some human malignancies, but has been poorly documented in breast carcinomas

METHODS: The prognostic significance of c Kit in a series of 924 breast carcinomas (mean follow-up, 79 months) was investigated using standardised high-throughput quantitative densitometry of immunohistochemical precipitates in tissue microarrays.

RESULTS: c Kit was expressed in 14.7\% breast carcinomas (and in 42 out of 586 node-negative tumours). In univariate analysis, (log-rank test) the score of $c$ Kit expression correlated with poor patient outcome $P=0.02$ and particularly in node-negative cases $(P=0.002)$. In multivariate Cox analysis, $c$ Kit was an indicator of metastasis independent of 25 other concomitantly evaluated markers of prognosis. Logistic regression showed that c Kit ranked 10 out of $25(P=0.04 \mathrm{I})$, and was included in a 10 -marker signature that allowed $79.2 \%$ of the patients to be correctly classified in the metastatic or metastasis-free categories independently of hormone receptors and HER-2 status. Interestingly, c Kit was also a significant predictor of metastasis in node-negative tumours (2 out of 25 ranking, $P<0.000 \mathrm{I})$ and included in a six-marker signature of prognosis, correctly classifying $88.6 \%$ of the patients $(P<0.000 \mathrm{I})$. CONCLUSION: We concluded that, as assessed by quantitative immunohistochemistry, $\mathrm{c}$ Kit is an independent prognostic indicator that could also potentially serve as a target for specific therapy in breast carcinomas.

British Journal of Cancer (2009) I 0 I, 48-54. doi:I0.1038/sj.bjc.6605 II 3 www.bjcancer.com

Published online 9 June 2009

(c) 2009 Cancer Research UK

Keywords: c Kit; quantitative immunohistochemistry; breast carcinomas

As a transmembrane tyrosine kinase, $c$ Kit plays a physiological role in the development of several cell types including haematopoietic cells, germ cells and melanocytes (Miettinen and Lasota, 2005). In breast tissue, c Kit is found in normal epithelium and non-neoplastic breast lesions, suggesting a role in the maintenance of breast glandular epithelium (Matsuda et al, 1993; Miettinen and Lasota, 2005).

Published data show a low prevalence of c Kit expression in breast carcinomas, ranging from 1 to $13 \%$ (Tsuura et al, 2002; Nielsen et al, 2004; Simon et al, 2004; Tsuda et al, 2005; Reis-Filho and Tutt, 2008), and in one report up to $25 \%$ (Tsutsui et al, 2006). The variations in expression in previous immunohistochemical studies can be explained by a lack of standardisation of procedures, particularly of quantification of immunostaining.

Therefore, it is clear that, first, expression of c Kit needs to be evaluated with a high-throughput standardised procedure in large series to determine the real prevalence and the clinical relevance of its identification in individual breast carcinomas. Second, correlation of the levels of c Kit expression with patients' outcome will show its actual prognostic value.

\footnotetext{
*Correspondence: Professor C Charpin, Hôpital Nord - Service d'Anatomie et de Cytologie Pathologiques, Chemin des Bourrelly, Marseille, 13915 Cedex 20, France; E-mail: colette.charpin@ap-hm.fr Revised 27 April 2009; accepted 29 April 2009; published online 9 June 2009
}

In the present immunohistochemical study of 924 invasive breast carcinomas, we investigated $c$ Kit expression within tissue microarrays (TMA), quantified by densitometry on digitised microscopic images using an image analysis device and dedicated software. The expression of c Kit was correlated with (1) patients' outcome (mean follow-up, 79 months) and (2) that of 25 other prognostic markers, with particular attention to node-negative tumours.

\section{MATERIALS AND METHODS}

\section{Patients}

The participants were a consecutive series of 1200 patients with invasive breast carcinomas who were operated on from 1995 to 2002 (mean follow-up, 79 months) in the same department at the Hôpital Conception, Marseille. Surgery was in all cases the first treatment $(\mathrm{PB})$. For this first step of treatment, patient management was handled by the same group of surgeons and by three senior pathologists (CC, SG, LA). Conservative treatment, mastectomy and node resection (complete or sentinel) were applied according to the current European recommendations. Likewise, radiotherapy, chemotherapy and hormone therapy were applied according to criteria currently used at that time.

Analysis of the distribution of the series by age, histological type and grade, and nodal status before TMA construction revealed the 
usual distribution of breast carcinomas and no bias in tumour selection, as compared with literature data. Owing to the technical difficulties in carrying out immunocytochemical tests on many serial paraffin sections of a TMA to evaluate the 35 different markers (Table 1), complete data for all markers were finally obtained for only 924 patients out of the initial series of 1200 .

The 2005 follow-up data in clinical records showed that 181 out of 924 patients had metastatic tumours.

Our study focused mainly on correlation of quantitative immunohistochemical data with patients' outcome. Current histoprognostic criteria on $\mathrm{H}$ and $\mathrm{E}$ staining were not retained for statistical analysis, mainly to limit the burden of data and also to focus the statistical analysis on continuous variables homogeneously obtained by (numerical) densitometric measurement of immunoprecipitates with the image analysis device.

\section{Tissue}

Tissue samples were all archival material taken from consecutive surgical specimens after formalin fixation and paraffin embedded blocks. Attention was paid to optimal consistent tissue-handling procedures, including fast immersion in buffered formalin in an appropriate container by pathologists or by nurses trained in the procedure. Tumour fragments were large $(5 \mathrm{~mm})$ and thick enough $(3 \mathrm{~mm})$ to allow further TMA construction. Duration of fixation was $24 \mathrm{~h}$ for smaller samples $(<5 \mathrm{~cm})$ and $48 \mathrm{~h}$ for larger ones, to improve formalin penetration, before specimen dissection at room temperature. After fixation, paraffin pre-embedding and embedding were carried out with currently available automated devices of the same brand.

Table I Sources of antibodies for immunodetection in tissue microarrays

\begin{tabular}{|c|c|c|c|}
\hline Antibody & Supplier & Source & Clone \\
\hline CDII7 (c-Kit) & Dako & Rpab & \\
\hline E-Cadherin & Zymed & Mmab & $4 \mathrm{~A} 2 \mathrm{C} 7$ \\
\hline CAIX & Abcam & Rpab & \\
\hline Cytokeratin 903 & Dako & Mmab & 34BEI2 \\
\hline P63 & Dako & Mmab & $4 \mathrm{~A} 4$ \\
\hline FYN & Abcam & Mmab & IS \\
\hline SHARP 2 & Abcam & Rpab & \\
\hline P2IWafl-Cip I & Cell Signaling & Mmab & DCS60 \\
\hline P27 Kipl & Cell Signaling & Rpab & \\
\hline P38 MAP kinase & Cell Signaling & Rpab & \\
\hline FAK & Cell Signaling & Rpab & \\
\hline STAT-I & Cell Signaling & Mmab & $9 \mathrm{H}_{2}$ \\
\hline EGFR & Ventana & Mmab & $3 \mathrm{C} 6$ \\
\hline Phospho-MAPKAPK-2 & Cell Signaling & Rmab & (Thr334) \\
\hline Cytokeratin 19 & Dako & Mmab & BAI7 \\
\hline Vimentin & Immunotech & Mmab & V9 \\
\hline CD34 & Dako & Mmab & QBEnd-IO \\
\hline CDIO & Novocastra & Mmab & $56 C 6$ \\
\hline STAT-3 & Cell Signaling & RMab & Tyr 705 D3A7 \\
\hline Cytokeratin 17 & Dako & Mmab & E3 \\
\hline Moesin I & Biomeda & Mmab & $38 / 87$ \\
\hline CD44v6 & Novocastra & Mmab & VFF-7 \\
\hline Ezrin (p8 I,80k,cytovillin) & Neomarkers & Mmab & $3 \mathrm{C} 12$ \\
\hline FGFR-I Flg (C-15) & Santa Cruz & Rpab & \\
\hline P16 & Neomarkers & Mmab & $\mathrm{Ab} 7(16 \mathrm{PO})$ \\
\hline P53 & Dako & Mmab & DO-7 \\
\hline $\mathrm{Bc} 12$ & Dako & Mmab & 124 \\
\hline CDI46 & Novocastra & Mmab & NI238 \\
\hline Caveolin I & Santa Cruz & Rpab & \\
\hline c-Met & Chemicon/Abcys & Mmab & 4AT44 \\
\hline JAK I & Cell Signaling & Rpab & \\
\hline Cytokeratins 5-6 & Dako & Mmab & D5/I6B4 \\
\hline
\end{tabular}

Abbreviations: $M m a b=$ mouse monoclonal antibody; $\mathrm{Rpab}=$ rabbit polyclonal antibody.
Paraffin blocks were stored in the same room, in which temperature was maintained at $20^{\circ} \mathrm{C}$ before TMA construction.

\section{TMA construction}

The procedure for construction of TMAs was as previously described (Garcia et al, 2007a,b). Briefly, cores were punched from the selected 1200 paraffin blocks (from 1200 patients), distributed in six new blocks including two cores for each tumour (200 cases per block, a total of 2400 cores) of $0.6-\mathrm{mm}$ diameter. All the new blocks (TMAs) were stored at $4{ }^{\circ} \mathrm{C}$, before sections $4-\mu \mathrm{m}$ thick were prepared for each marker to be examined by immunohistochemistry.

\section{Immunohistochemistry}

Serial tissue sections were prepared and stored at $4^{\circ} \mathrm{C}$ for $24 \mathrm{~h}$ before immunohistochemical processing, as previously reported (Garcia et al, 2007a,b). Immunoperoxidase procedure was performed using an automated Ventana Benchmark XT device and Ventana Kits (Ventana, Strasbourg, Illkirch, France).

Markers were detected using commercially documented antibodies (Table 1). Dilutions of antibodies were determined by pre-screening on the usual full $4-\mu \mathrm{m}$ thick sections before use on TMA sections.

\section{Image analysis}

Automated densitometric measurements of immunoprecipitates in cores were made for each marker antibody in each core individually identified after digitisation and image cropping of the slides, as previously reported (Garcia et al, 2007a,b). Briefly, TMA analysis with a SAMBA 2050 automated device (SAMBA Technologies, Meylan/Grenoble and TRIBVN, Chatillon, France) (Charpin et al, 1997b, 1998a, b, 2004) was carried out according to the following protocol.

First, an image of the entire slide was built up using low-power magnification $(\times 2$, pixel dimension $3.7 \mu \mathrm{m})$. This image was made up of a mosaic of images acquired along a rectangular grid with contiguous fields. Second, the area of the slide containing the TMA cores was automatically delineated and scanned at higher magnification $(\times 20$, pixel dimension $7.4 \mu \mathrm{m})$. Third, after autofocusing, the images were acquired with an overlap greater than the largest mechanical positioning error. Using the image contents, a matching algorithm determined precisely the relative position of each image with respect to its neighbours. Calculated overlap was removed from images to produce a new set of higher-magnification images, thus covering precisely the cores of interest. A specially developed tool referred to as TMA crop (Plaisir, France) then allowed superposition of the TMA grid onto the reduced image and precise alignment of each node of the grid with the core location within the image. The final step was carried out automatically using the core image contents to ensure pixel precision of the match. From the images acquired with $\times 20$ magnification, a new set of images was next computed, one for each core. For colour analysis of the core images, the SAMBA 'immuno' software was applied as previously reported (Charpin et al, 1994, 1997a, b, 1998a, b, c, 2004; Garcia et al, $2007 \mathrm{a}, \mathrm{b})$ in the usual full-tissue sections.

In the present study, we correlated the patients' follow-up parameters with a quantitative score combining the surface stained and the intensity of staining (Garcia et al, 2007a,b) computed by the SAMBA 'immuno' software. The threshold for positive staining was determined according to a densitometric measurement of c Kit immunostaining in normal tissue run in the same batch as the TMAs. 


\section{Statistical analysis}

Immunohistochemical expression of each marker was first correlated with patients' disease-free survival using NCSS (www.ncss. com) and Statistica statistical software (www.statsoft.com).

When significant differences in mean expression were identified in patients with disease and without disease, the prognostic significance was determined by log-rank tests (Kaplan-Meier curves). The appropriate threshold of prognostic significance for a given marker was determined as previously recommended (Altman et al, 1994) and described (Charpin et al, 1994, 1997a, b, 1998a, b, c, 2004; Garcia et al, 2007a, b).

Logistic regression (with ROC curves) was then used to identify the combination of markers with the best sensitivity and specificity indicative of a proteomic signature of poor prognosis.

Finally, unsupervised hierarchical clustering of significant prognostic indicators in the overall series provided qualitative data to be compared with previously reported research results on relationships between these molecules, and on the role played by them in the process of cancer metastasis.
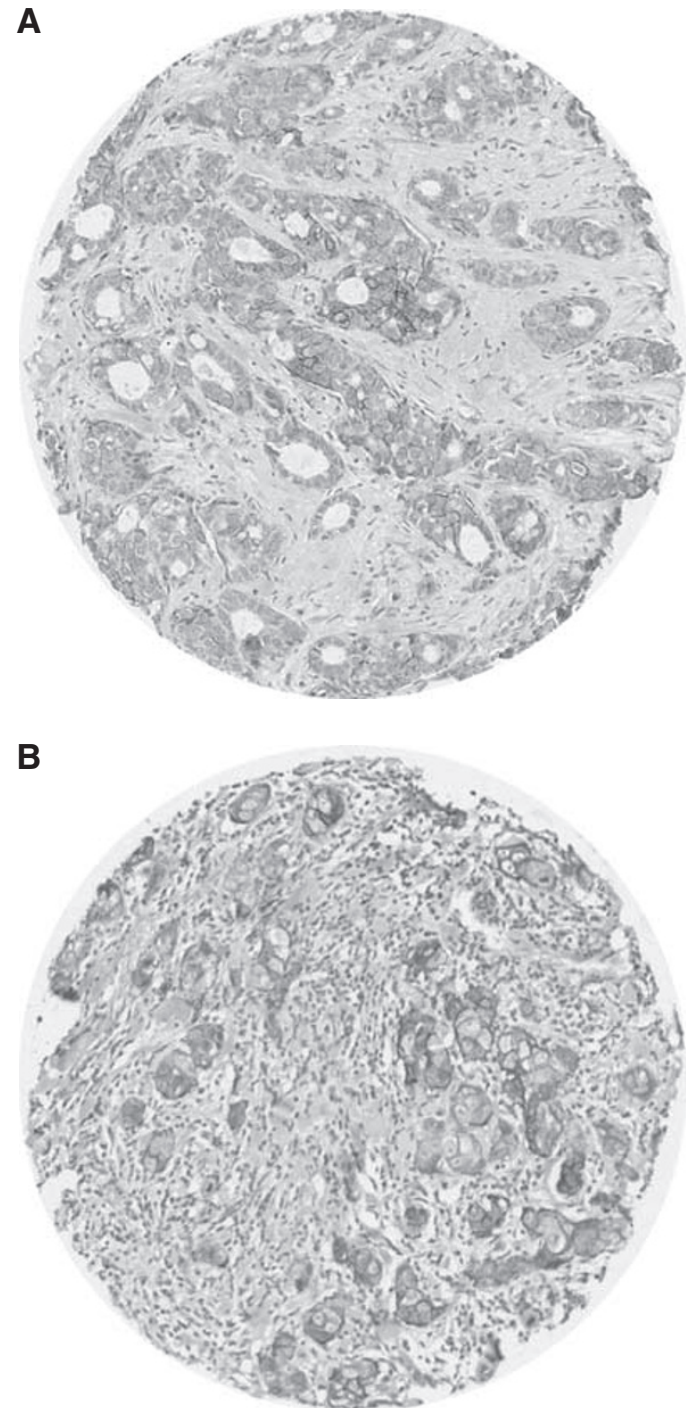

Figure I c Kit-positive immunostaining $(\mathbf{A})$ in grade 2 breast carcinoma and $(\mathbf{B})$ in grade 3 breast carcinomas: 'spots' corresponding to tumour cores measuring $0.6 \mathrm{~mm}$ in diameter lead to tissue microarray (TMA).

\section{RESULTS}

c Kit distribution in node-positive $(\mathrm{N}+)$ and node-negative $(\mathrm{N}-)$ tumours

The screening of spots by image analyser after TMA 'cropping' revealed that $135(14.7 \%)$ of all tumours were c Kit-positive, whereas among node-negative tumours, 42 out of $584(7.2 \%)$ and among node-positive tumours 71 out of 340 (21\%) were c Kitpositive.

Positive staining was observed in the cell membrane in normal breast and also in tumours, as shown in Figure 1.

The mean quantitative score for c Kit, automatically computed by the image analyser, was significantly higher $(P=0.0007)$ in tumours of patients with distant metastasis $(m=12.2$, s.d. $=4.3$; $n=181$ ) than in tumours of those lacking distant metastasis $(m=4.08$, s.d. $=1.2 ; n=743)$. Likewise, the mean score was higher $(P<0.0001)$ in node-positive tumours $(m=6.29$; s.d. $=2.11)$ than in node-negative tumours $(m=3.47$; s.d. $=0.63)$.

\section{Prognostic value of $c$ Kit in univariate analysis}

Comparison of c Kit expression and patients' outcome using log-rank tests showed that $\mathrm{c}$ Kit was a significant prognostic indicator in all subgroups $(P=0.02)$ and in node-negative patients $(P=0.002)$ (Figure 2).
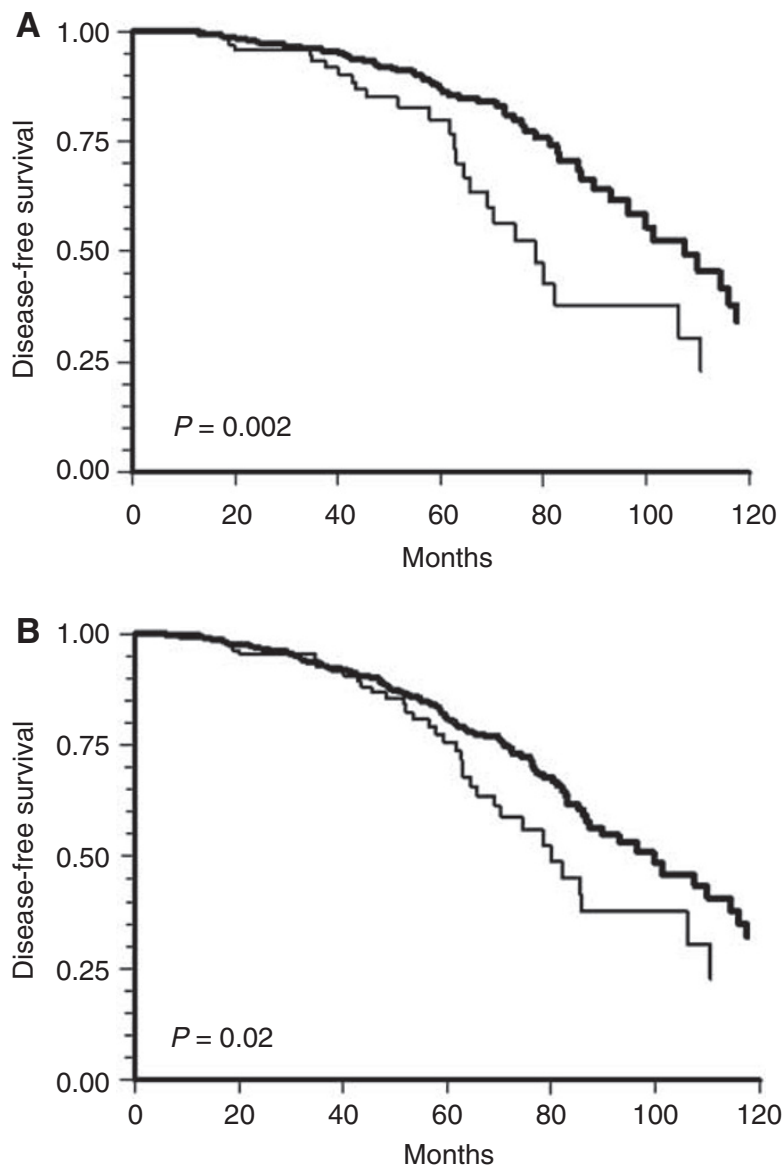

Figure 2 Kaplan-Meier curve showing significant correlation of c Kit densitometry and development of distant metastases (mean follow-up $(P=0.02$ and $P=0.002$ respectively) 79 months) in (A) 924 breast carcinoma and (B) 586 node-negative tumours. 
A

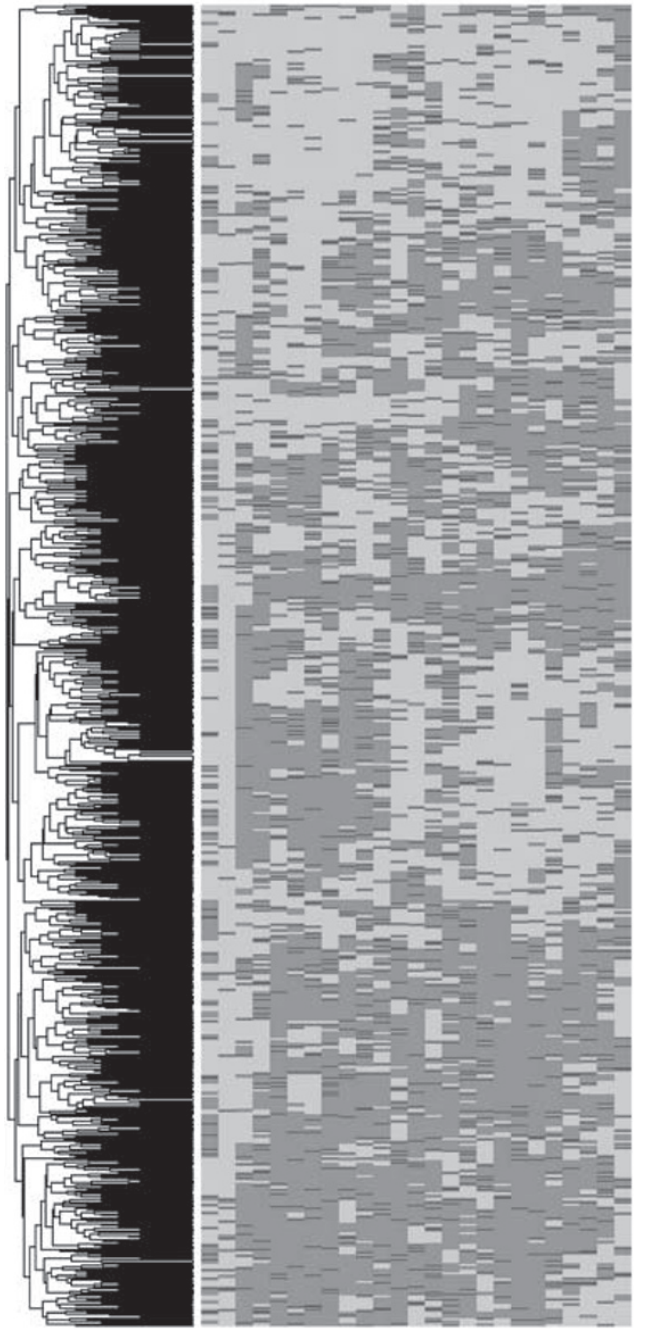

B

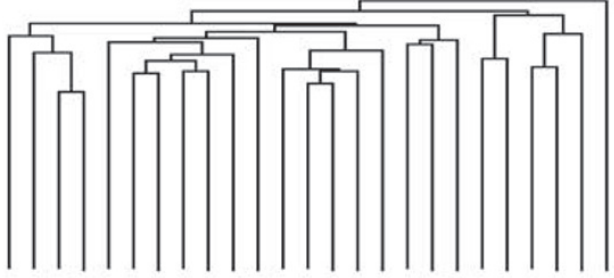

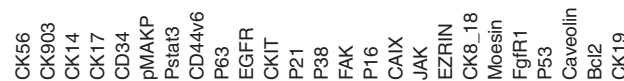

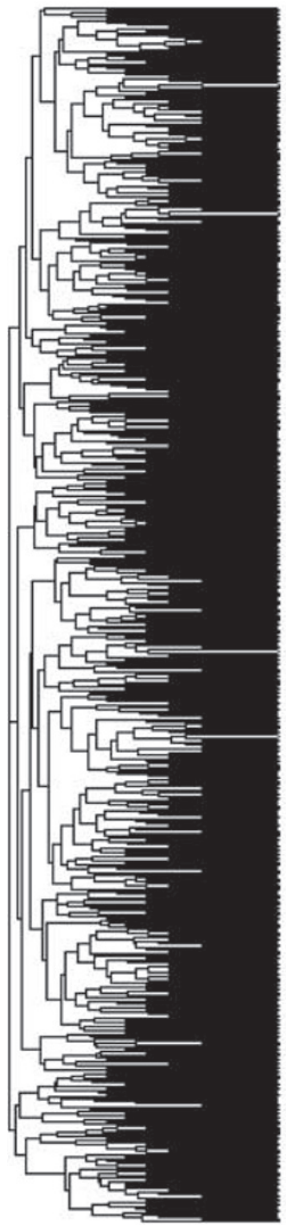

Figure 3 Unsupervised hierarchical clustering of 25 (individually prognostic significant often log-rank tests) markers evaluated by quantitative immunohistochemical (image analysis/densitometry) expression, on tissue microarray (TMA) in (A) 924 breast carcinomas and (B) 586 node-negative carcinomas.

\section{Multivariate analysis}

Multivariate Cox analysis showed that $c$ Kit was an independent prognostic indicator when evaluated with 24 other prognostic indicators categorised as such, using the same quantitative procedure as that in univariate analysis (log-rank test, $P<0.01$ ), in the whole series of 924 tumours and in the 584 node-negative tumours.

Hierarchical unsupervised clustering (Figure 3) showed the relationship of $\mathrm{c}$ Kit with the other markers, independently of hormone receptor and HER-2 status.

To determine the prognostic value, the ranking of $c$ Kit was compared with that of 25 other markers in logistic regression in the series of 924 patients and in the 584 node-negative tumours, independently of ER, PR and HER-2 expression (Tables 2 and 3, Figures 4-7).
The first step of logistic regression in the series of 924 tumours showed that 10 out of the 25 markers tested allowed correct classification of $81.17 \%$ patients in both categories of good and poor prognosis (sensitivity $78.5 \%$, specificity $92.3 \%$, area under ROC curve 0.906), as shown in Figure 4, and the ranking of c Kit, based on odds ratio and $P$-value of deviance increase $(P=0.012)$, was 10 out of 25 (Table 2).

When a second regression step was assessed with the significant markers from the first step, including $\mathrm{c}$ Kit, the results showed that a slightly lower percentage of patients $(79.22 \%)$ were well classified (sensitivity $75.9 \%$, specificity $92.8 \%$, area under ROC curve 0.89) (Figure 5), as compared with the first regression step $(81.17 \%)$. All 10 markers including $c$ Kit remained highly significant for prognostic prediction of metastasis $(P$-value of deviance increase $=0.0411$ ). 
Table 2 Logistic regression of 25 immunohistochemical markers (quantitative score from image analysis) in tissue microarrays of 924 breast carcinomas

\begin{tabular}{|c|c|c|c|}
\hline & Marker & Odds ratio & $P$-value of deviance increase \\
\hline I & Moes & 0.6939 & 0.0000 \\
\hline 2 & P53 & 0.4091 & 0.0000 \\
\hline 3 & PMAPK & 1.4654 & 0.0000 \\
\hline 4 & P38 & 07979 & 0.0026 \\
\hline 5 & CalX & I.194I & 0.0041 \\
\hline 6 & CD44v6 & 1.1866 & 0.0071 \\
\hline 7 & CK903 & 0.8573 & 0.0191 \\
\hline 8 & P2I & 0.8577 & 0.0249 \\
\hline 9 & CAVEOL & 0.7986 & 0.0322 \\
\hline 10 & c kit & 0.8816 & 0.0411 \\
\hline || & CK5-6 & 1.1262 & 0.0654 \\
\hline 12 & JAK & 1.1110 & $0.07 \mid 1$ \\
\hline 13 & K8- 18 & 0.8925 & 0.0864 \\
\hline 14 & PI6 & 0.9078 & 0.1292 \\
\hline 15 & EGFR & 1.1024 & 0.1392 \\
\hline 16 & $\mathrm{KI} 4$ & 1.1062 & 0.1471 \\
\hline 17 & CD34 & 0.9292 & 0.2282 \\
\hline 18 & P63 & 1.0754 & 0.3291 \\
\hline 19 & FAK & 0.9400 & 0.3666 \\
\hline 20 & PSTAT3 & $0.947 \mid$ & 0.4750 \\
\hline 21 & $\mathrm{Bc} 2$ & 1.0452 & 0.4968 \\
\hline 22 & CK19 & 1.0413 & 0.5140 \\
\hline 23 & Ezrin & 0.9648 & 0.5737 \\
\hline 24 & FgFRI & 0.9792 & 0.7484 \\
\hline 25 & CKI7 & 0.9999 & 0.9982 \\
\hline
\end{tabular}

Bold digits signify markers found to be prognostically significant.

Table 3 Logistic regression of 25 immunohistochemical markers (quantitative score from image analysis) in tissue microarrays of 586 node-negative breast carcinomas

\begin{tabular}{llcc}
\hline & Markers & Odds ratio & P-value of deviance increase \\
\hline 1 & P53 & $\mathbf{0 . 3 2 9 0}$ & $<\mathbf{0 . 0 0 0 0}$ \\
2 & c kit & $\mathbf{0 . 6 1 9 0}$ & $<\mathbf{0 . 0 0 0 0}$ \\
3 & P21 & $\mathbf{0 . 6 1 9 0}$ & $\mathbf{0 . 0 0 0 1}$ \\
4 & CK903 & $\mathbf{0 . 7 3 3 1}$ & $\mathbf{0 . 0 0 8 2}$ \\
5 & Moes & $\mathbf{0 . 7 3 6 9}$ & $\mathbf{0 . 0 2 0 4}$ \\
6 & CKI7 & $\mathbf{1 . 3 5 2 9}$ & $\mathbf{0 . 0 2 1 7}$ \\
7 & EGFR & 1.2385 & 0.0553 \\
8 & CD44V6 & 1.3099 & 0.0609 \\
9 & BCI2 & 1.2337 & 0.0679 \\
10 & P38 & 1.2730 & 0.0793 \\
11 & PMAPK & 1.2851 & 0.0889 \\
12 & JAK & 1.1817 & 0.1144 \\
13 & FgFRI & 0.8399 & 0.1194 \\
14 & PI6 & 1.2008 & 0.1495 \\
15 & CalX & 1.1726 & 0.1766 \\
16 & CK8-18 & 1.1561 & 0.2086 \\
17 & FAK & 0.8568 & 0.2249 \\
18 & Ezrin & 1.0852 & 0.4662 \\
19 & P63 & 1.0564 & 0.6560 \\
20 & CKI4 & 1.0375 & 0.7727 \\
21 & PSTAT3 & 1.0262 & 0.8695 \\
22 & CKI9 & 1.0157 & 0.8876 \\
23 & KER56 & 1.0121 & 0.9157 \\
24 & CAVEOL & 1.0286 & 0.9223 \\
25 & CD34 & 0.9908 & 0.9341 \\
\hline
\end{tabular}

Bold digits signify markers found to be prognostically significant.

Interestingly, when the 584 node-negative tumours were considered, the first-step regression showed that $c$ Kit ranked second among the six-marker signature (Table 3) that correctly classified $80.95 \%$ of the patients (sensitivity $80.4 \%$, specificity

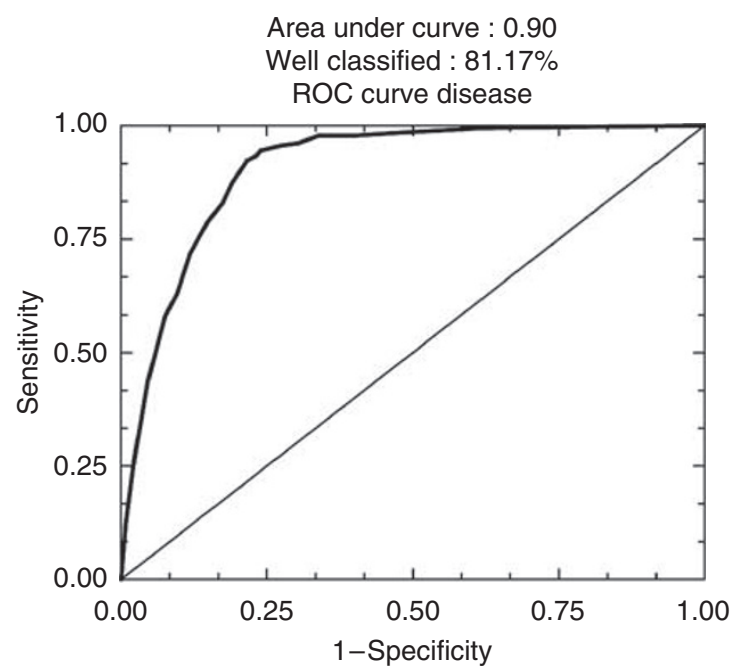

Figure 4 ROC curves after logistic regression of quantitative immunohistochemical expression of 25 prognostic markers in breast carcinomas on tissue microarray (TMA): in whole series $(n=924)(81.17 \%$ of wellclassified patients), first step of regression.

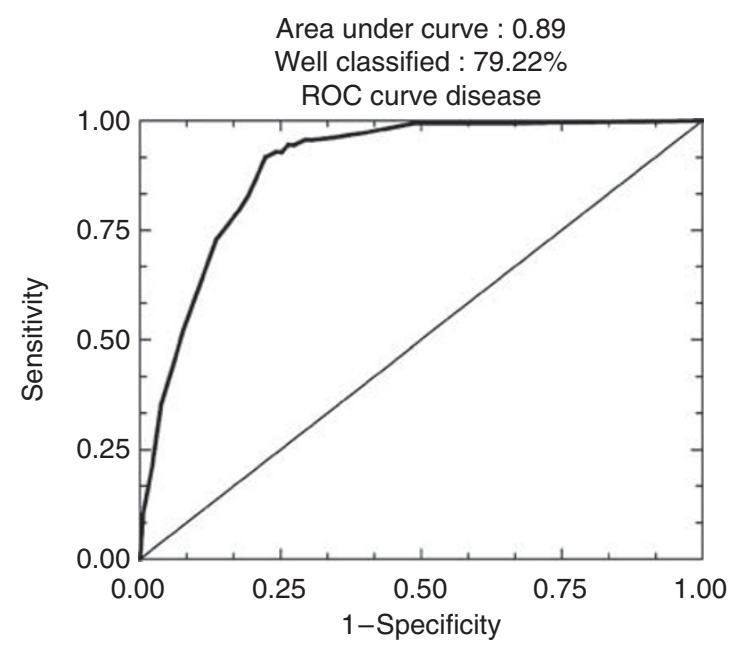

Figure 5 ROC curves after logistic regression of quantitative immunohistochemical expression of 25 prognostic markers in breast carcinomas on tissue microarray (TMA): second step of regression (79.22\% of wellclassified patients).

$83.8 \%$, area under ROC curve 0.960 ) (Figure 6) in the metastatic or metastasis-free subsets. Moreover, c Kit remained very prognostically significant $(P<0.0001)$ along with six others. The ranking of c Kit was 2 out of 25, based on odd ratios and $P$-values of deviance increase (Table 3 ).

Finally, when a second step of regression was carried out in this node-negative subset with only the six most prognostically significant markers out of 25, patients were well classified in $88.6 \%$ (sensitivity $90.3 \%$, specificity $86.5 \%$; area under ROC curve 0.96; Figure 7) and c Kit remained clearly a prognostic predictor and still ranked 2 out of 6.

\section{DISCUSSION}

Breast cancer is a heterogeneous disease, encompassing a number of distinct biological entities that are associated with specific 


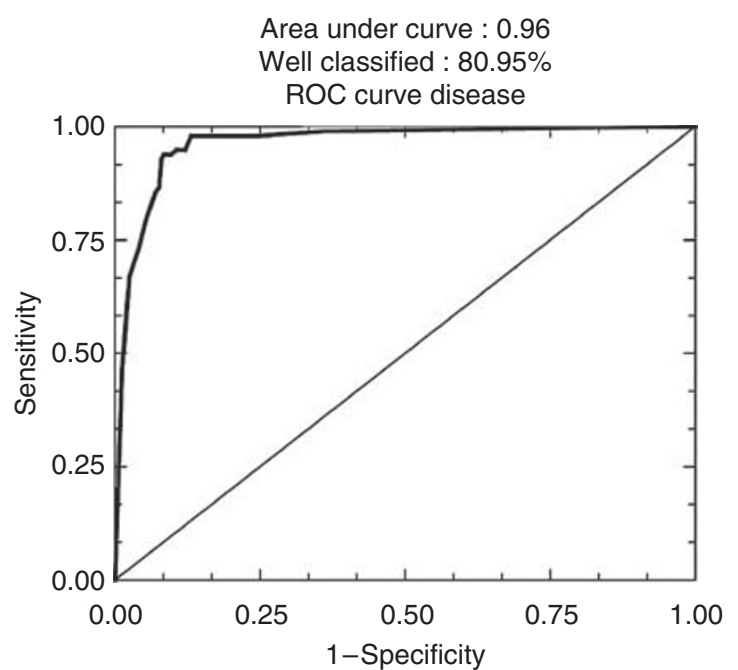

Figure 6 ROC curves after logistic regression of quantitative immunohistochemical expression of 25 prognostic markers in breast carcinomas on tissue microarray (TMA): in node-negative carcinomas $(n=586)$ first step (80.95\% of well-classified patients).

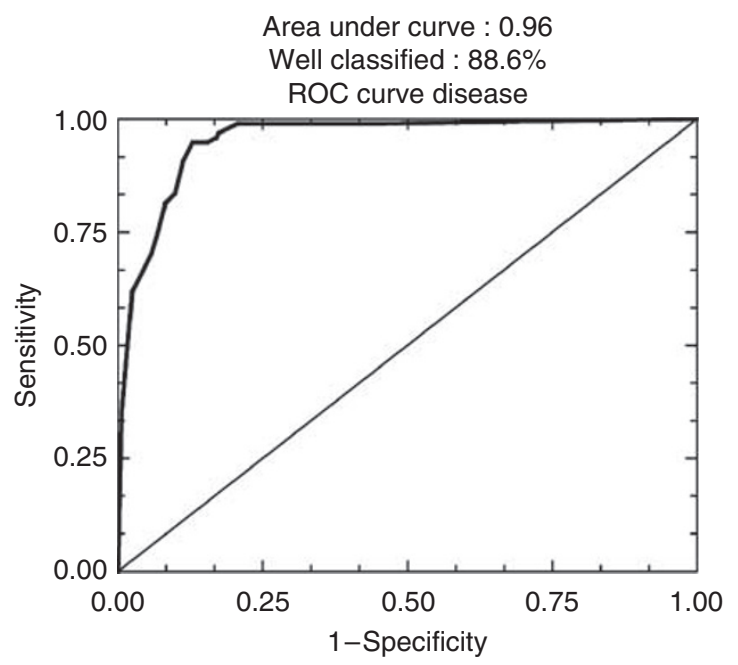

Figure 7 ROC curves after logistic regression of quantitative immunohistochemical expression of 25 prognostic markers in breast carcinomas on tissue microarray (TMA): second step (88.6\% of well-classified patients).

morphological and immunohistochemical features and clinical behaviour (Simpson et al, 2005). Despite this morphological heterogeneity, however, patients can practically be classified into three main groups for management and therapy according to: (1) hormone receptor (ER, PR) positivity, (2) presence of Her-2 neu (c-erb B2) amplification or (3) absence of these two characteristics. In the latter group of patients lacking specific targets for hormone and trastuzumab therapy, there is a need to identify new targets for tailored treatments. Genome microarray analysis (Perou et al, 2000; Sorlie et al, 2003) and expression profiling (Reis-Filho et al, 2006) have recently been used to characterise five groups of breast cancers that can also be identified by immunohistochemical screening. In particular, for the triple-negative (ER-PgR-HERnegative) tumours, this deeper molecular insight into tumour characterisation should allow new targets for tailored therapies to be identified. In this regard, the inhibition of $c$ Kit gene expression by imatinib (STI 571, Glivec), which was initially shown to be effective in the treatment of chronic myeloid leukaemia (Kantarjian et al, 2002), has more recently been found effective also against c Kit-positive gastrointestinal stroma tumours (GIST) (Miettinen et al, 2001), suggesting that other c Kit-positive tumours, in particular breast carcinomas, may respond to imatinib therapy.

In breast cancer, expression of c Kit is reported to be reduced and detected in 1-13\% of tumours (Tsuura et al, 2002; Nielsen et al, 2004; Simon et al, 2004; Tsuda et al, 2005; Reis-Filho and Tutt, 2008). Our results are close to the upper range of previous reports, with $14.7 \%$ of positive tumours in our series. However, some conflicting results have also recently been reported showing a decrease of cKit expression in advanced stage and poor prognosis in breast cancer (Tsutsui et al, 2006). The variations in the literature data probably result from the diversity of immunodetection procedures used and also from the lack of results quantification. In this respect, automated quantification of immunoreactions in sections of hundreds of 0.6-micron-thick micro-biopsy cores, using dedicated software to measure extents of staining by densitometry after 'cropping' on digitised microscopic images of immunostained TMA, provides a time- and cost-effective, reproducible and accurate means of evaluation, particularly in comparison with approximate and subjective semi-quantitative methods.

Literature data show that $\mathrm{c}$ Kit expression in breast cancer is more common in basal-like carcinomas (31\%), medullary (19\%), grade $3(24 \%)$ and metaplastic (57\%) carcinomas (Nielsen et al, 2004; Reis-Filho and Tutt, 2008). In our study, c Kit staining was also found to be significantly more highly expressed in more aggressive, node-positive tumours than in node-negative carcinomas (results not shown).

Our results show that $\mathrm{c}$ Kit is part of an immunohistochemical signature that permits correct classification of $81.17 \%$ patients in the metastatic or metastasis-free categories (mean follow-up 79 months), and $80.95 \%$ of node-negative patients. Patients could thus be selected for more aggressive therapy according to evidence from analysis of this immunohistochemical signature at the time of diagnosis, which can be carried out with only a small amount of fixed and paraffin-embedded tissue (7 to 104 -micron-thick tissue sections) remaining in blocks after microscopic diagnosis and pTN staging. Moreover, this procedure is significantly less timeconsuming, as well as cheaper to carry out, than molecular (genome and transcriptome) profiling, so that the results can be available within the same timescale as the pathological report. Thus, standardised quantification of this immunohistochemical signature including cKit (using automated image analysis) could be suitable to examine individual tumours in routine clinicopathological practice, in both node-positive and -negative breast cancers.

Likewise, the prognostic significance of $c$ Kit expression in tumours, in conjunction with the other markers of the signature established by logistic regression, may allow selection of patients for more aggressive therapy, particularly with node-negative tumours. Also, c Kit expressed in the $14.7 \%$ of tumours (included in our series) that were positive may serve as a target for specific therapy with imatinib. However, in contrast to GIST, for which imatinib has proved to be an efficient tailored therapy, experience with imatinib therapy in breast cancer is limited. A trial conducted by Modi et al (2005) did not establish clinical benefit. However, in that study, no $c$ Kit expression was detected in 8 out of the 11 patients enroled with available tissue. In another pilot study, 9 out of 10 patients enroled with moderate expression of cKit in tumours were partially responsive to imatinib associated with aromatase inhibitors (Chow et al, 2008).

In GIST, accumulation of $\mathrm{c}$ Kit is usually related to activating mutations. In breast cancer, no mutation has so far been found, though reports referred to very short patient series $(n=10)$ (Simon et al, 2004). The relationships of c Kit expression and 
mutations, and patients' response to imatinib-tailored therapy deserve further investigation in conjunction with clinical trials to gain deeper insight into pathways of $\mathrm{c}$ Kit regulation and signalling in breast cancer.

In conclusion, our study shows that, as assessed with our highthroughput quantitative immunohistochemical procedure in TMAs from 924 breast cancers, c Kit was expressed in $14.7 \%$ of patients and was predictive of patients' outcome, and also in nodenegative subsets. Evaluation of c Kit concomitantly with 10 or 6 other prognostic markers by the same method provides a costeffective procedure permitting the correct classification of 81.17 $88.6 \%$ of the patients into the metastatic and metastasis-free categories, independent of hormone receptor and HER-2 status, and may be useful in selecting node-negative patients for more aggressive therapy. Finally, for tumours expressing cKit, patients should potentially benefit from tailored therapy with imatinib, in a similar manner to the use of trastuzumab to treat tumours that strongly overexpress HER-2. However, deeper insight into the mechanisms of $c$ Kit downregulation and clinical trials are required to show the relevance of this tailored therapy in breast cancer, as seen with other malignancies such as GIST.

\section{ACKNOWLEDGEMENTS}

This study was supported by a grant from the Institut National contre le Cancer/Canceropole Provence Alpes Côte d'Azur AP-HM. We are grateful to Roche for supporting a Master's project (V Secq).

\section{REFERENCES}

Altman DG, Lausen B, Sauerbrei W, Schumacher M (1994) Dangers of using 'optimal' cutpoints in the evaluation of prognostic factors. J Natl Cancer Inst 86: 829-835

Charpin C, Dales JP, Garcia S, Carpentier S, Djemli A, Andrac L, Lavaut MN, Allasia C, Bonnier P (2004) Tumor neoangiogenesis by CD31 and CD105 expression evaluation in breast carcinoma tissue microarrays. Clin Cancer Res 10: 5815-5819

Charpin C, Garcia S, Bonnier P, Martini F, Andrac L, Choux R, Lavaut MN, Allasia C (1998a) Reduced E-cadherin immunohistochemical expression in node-negative breast carcinomas correlates with 10 -year survival. Am J Clin Pathol 109: $431-438$

Charpin C, Garcia S, Bonnier P, Martini F, Andrac L, Horschowski N, Lavaut MN, Allasia C (1998b) Prognostic significance of Nm23/NDPK expression in breast carcinoma, assessed on 10-year follow-up by automated and quantitative immunocytochemical assays. J Pathol 184: 401-407

Charpin C, Garcia S, Bonnier P, Martini F, Andrac L, Horschowski N, Lavaut MN, Allasia C (1998c) bcl-2 automated and quantitative immunocytochemical assays in breast carcinomas: correlation with 10-year follow-up. J Clin Oncol 16: 2025-2031

Charpin C, Garcia S, Bouvier C, Devictor B, Andrac L, Choux R, Lavaut MN, Allasia C (1997a) Automated and quantitative immunocytochemical assays of CD44v6 in breast carcinomas. Hum Pathol 28: 289-296

Charpin C, Garcia S, Bouvier C, Martini F, Andrac L, Bonnier P, Lavaut MN, Allasia C (1997b) CD31/PECAM automated and quantitative immunocytochemical assays in breast carcinomas: correlation with patient follow-up. Am J Clin Pathol 107: 534-541

Charpin C, Vielh P, Duffaud F, Devictor B, Andrac L, Lavaut MN, Allasia C, Horschowski N, Piana L (1994) Quantitative immunocytochemical assays of P-glycoprotein in breast carcinomas: correlation to messenger RNA expression and to immunohistochemical prognostic indicators. $\mathrm{J} \mathrm{Natl}$ Cancer Inst 86: 1539-1545

Chow LW, Yip AY, Loo WT, Toi M (2008) Evaluation of neoadjuvant inhibition of aromatase activity and signal transduction in breast cancer. Cancer Lett 262: $232-238$

Garcia S, Dales JP, Charafe-Jauffret E, Carpentier-Meunier S, Andrac-Meyer L, Jacquemier J, Andonian C, Lavaut MN, Allasia C, Bonnier P, Charpin C (2007a) Overexpression of c-Met and of the transducers PI3K, FAK and JAK in breast carcinomas correlates with shorter survival and neoangiogenesis. Int J Oncol 31: 49-58

Garcia S, Dales JP, Charafe-Jauffret E, Carpentier-Meunier S, Andrac-Meyer L, Jacquemier J, Andonian C, Lavaut MN, Allasia C, Bonnier P, Charpin C (2007b) Poor prognosis in breast carcinomas correlates with increased expression of targetable CD146 and c-Met and with proteomic basal-like phenotype. Hum Pathol 38: 830-841

Kantarjian H, Sawyers C, Hochhaus A, Guilhot F, Schiffer C, GambacortiPasserini C, Niederwieser D, Resta D, Capdeville R, Zoellner U, Talpaz M, Druker B, Goldman J, O’Brien SG, Russell N, Fischer T, Ottmann O, Cony-Makhoul P, Facon T, Stone R, Miller C, Tallman M, Brown R, Schuster M, Loughran T, Gratwohl A, Mandelli F, Saglio G, Lazzarino M, Russo D, Baccarani M, Morra E (2002) Hematologic and cytogenetic responses to imatinib mesylate in chronic myelogenous leukemia. $N$ Engl J Med 346: $645-652$
Matsuda R, Takahashi T, Nakamura S, Sekido Y, Nishida K, Seto M, Seito T, Sugiura T, Ariyoshi Y, Takahashi T, Ueda R (1993) Expression of the $\mathrm{c}$-kit protein in human solid tumors and in corresponding fetal and adult normal tissues. Am J Pathol 142: 339-346

Miettinen M, Furlong M, Sarlomo-Rikala M, Burke A, Sobin LH, Lasota J (2001) Gastrointestinal stromal tumors, intramural leiomyomas, and leiomyosarcomas in the rectum and anus: a clinicopathologic, immunohistochemical, and molecular genetic study of 144 cases. Am J Surg Pathol 25: $1121-1133$

Miettinen M, Lasota J (2005) KIT (CD117): a review on expression in normal and neoplastic tissues, and mutations and their clinicopathologic correlation. Appl Immunohistochem Mol Morphol 13: 205-220

Modi S, Seidman AD, Dickler M, Moasser M, D'Andrea G, Moynahan ME, Menell J, Panageas KS, Tan LK, Norton L, Hudis CA (2005) A phase II trial of imatinib mesylate monotherapy in patients with metastatic breast cancer. Breast Cancer Res Treat 90: 157-163

Nielsen TO, Hsu FD, Jensen K, Cheang M, Karaca G, Hu Z, HernandezBoussard T, Livasy C, Cowan D, Dressler L, Akslen LA, Ragaz J, Gown AM, Gilks CB, van de Rijn M, Perou CM (2004) Immunohistochemical and clinical characterization of the basal-like subtype of invasive breast carcinoma. Clin Cancer Res 10: 5367-5374

Perou CM, Sorlie T, Eisen MB, van de Rijn M, Jeffrey SS, Rees CA, Pollack JR, Ross DT, Johnsen H, Akslen LA, Fluge O, Pergamenschikov A, Williams C, Zhu SX, Lonning PE, Borresen-Dale AL, Brown PO, Botstein D (2000) Molecular portraits of human breast tumours. Nature 406: $747-752$

Reis-Filho JS, Tutt AN (2008) Triple negative tumours: a critical review. Histopathology 52: 108-118

Reis-Filho JS, Westbury C, Pierga JY (2006) The impact of expression profiling on prognostic and predictive testing in breast cancer. J Clin Pathol 59: 225-231

Simon R, Panussis S, Maurer R, Spichtin H, Glatz K, Tapia C, Mirlacher M, Rufle A, Torhorst J, Sauter G (2004) KIT (CD117)-positive breast cancers are infrequent and lack KIT gene mutations. Clin Cancer Res 10: 178-183

Simpson PT, Reis-Filho JS, Gale T, Lakhani SR (2005) Molecular evolution of breast cancer. J Pathol 205: 248-254

Sorlie T, Tibshirani R, Parker J, Hastie T, Marron JS, Nobel A, Deng S, Johnsen H, Pesich R, Geisler S, Demeter J, Perou CM, Lonning PE, Brown PO, Borresen-Dale AL, Botstein D (2003) Repeated observation of breast tumor subtypes in independent gene expression data sets. Proc Natl Acad Sci USA 100: 8418-8423

Tsuda H, Morita D, Kimura M, Shinto E, Ohtsuka Y, Matsubara O, Inazawa J, Tamaki K, Mochizuki H, Tamai S, Hiraide H (2005) Correlation of KIT and EGFR overexpression with invasive ductal breast carcinoma of the solid-tubular subtype, nuclear grade 3, and mesenchymal or myoepithelial differentiation. Cancer Sci 96: 48-53

Tsutsui S, Yasuda K, Suzuki K, Takeuchi H, Nishizaki T, Higashi H, Era S (2006) A loss of c-kit expression is associated with an advanced stage and poor prognosis in breast cancer. Br J Cancer 94: 1874-1878

Tsuura Y, Suzuki T, Honma K, Sano M (2002) Expression of c-kit protein in proliferative lesions of human breast: sexual difference and close association with phosphotyrosine status. J Cancer Res Clin Oncol 128: $239-246$ 